\title{
Osteoblastic Rimming Present
}

National Cancer Institute

\section{Source}

National Cancer Institute. Osteoblastic Rimming Present. NCI Thesaurus. Code C83005.

A morphologic finding indicating the presence of bony trabeculae rimmed with osteoblasts. 